\title{
A Routing Algorithm for Mobile Multiple Sinks in Large-Scale Wireless Sensor Networks
}

\author{
Mujdat Soyturk, Turgay Altilar \\ Istanbul Technical University, Department of Computer Engineering \\ Maslak, TR-34469, Istanbul, Turkey \\ msoyturk@itu.edu.tr, altilar@itu.edu.tr
}

\begin{abstract}
In wireless sensor networks, energy-aware and networklifetime prolonging techniques are proposed, as the energy becomes the most valuable resource. Most of these techniques use complex calculations failing to be easy to implement. Another approach is to use mobile-sink node to enhance the performance metrics. However, these techniques are not scalable to large scale networks. Scalability of a routing protocol for wireless sensor networks is mainly affected by the topological changes and by the number of sensor nodes in the network. In large scale networks, multiple-sinks (gateways) should be used to provide scalability. In this paper, we propose that mobile multiple sink usage enhances the energy-related performance metrics in large scale networks. We use the Stateless Weight Routing with Multiple Sinks (MS-SWR) for routing. Mobile sink nodes are used to enhance the performance metrics. The performance evaluation of the proposed technique shows that mobile sink usage in MS-SWR is scalable for large scale wireless sensor networks with multiple sinks.
\end{abstract}

\section{INTRODUCTION}

In wireless sensor networks, energy becomes one of the most valuable resources, as the network size increases. There are energy aware protocols in the literature, generally using multi-hop paths to use the energy more efficiently. However, increase in the hop number between the source and the destination nodes bears some issues that must be considered [1], [2]. First, nodes close to the sink deplete their energies quickly; leaving the sink unreachable and the system into offstate [3]. Secondly, increase in the hop-number cause more nodes to buffer the packet on-the-route, causing a processing overhead and delay at nodes. Processing overhead and buffer fill-up may cause the packets to be dropped. On the other hand, delay at nodes challenges with the real-time requirements of the system [1]. There are studies which utilize the advantage of mobile sinks to overcome such problems. Generally, a mobile sink is used to prolong the lifetime of the network and enhance performance metrics. In [4], sink node is repositioned to enhance the performance metrics. Other related studies [5]-[9] are proposed to prolong the lifetime of the network or to reduce the energy consumption in routing. However, as the network size grows, the length of the constructed paths will increase, causing the problem described above more challenging. On the other hand, the energy consumption will not be efficient anymore. The delay will increase, and the packets will be dropped. Packet drops will cause retransmissions, which increase the delay excessively.

Multiple sinks (multi-sink) usage appears as a solution for large scale networks [1], [3]. However, deploying more sink nodes does not solve the problem directly and evenly. Energy-efficient protocols should be adapted for the multisink networks. However, the protocol in use may not be energy-efficient anymore in large scale networks due to increase in the number of nodes. Related studies for multisink sensor networks are in [1]-[3], [10]-[14]. In this paper, we show that mobile multiple sink usage enhances the energy-related performance metrics. The proposed algorithm is based on the data flow approach proposed in [14] and [15]. Mobility of the sink nodes enhances the performance of MSSWR protocol due to reduction of transmissions in data flow to the sink.

In the next section, we review the related works. We give the mobility approaches for the multiple sinks in section 3 . Performance evaluations are given in Section 4. In the last section we conclude the paper.

\section{RELATED WORK}

Studies using mobile sink node generally attempt to prolong the lifetime of the network. Reference [5] proposes mobility patterns for the sink and takes the advantage of sink's mobility to prolong the lifetime of the network. In [6] and [7], it is proven that that mobile sink node improves the lifetime of the network. In order to maximize the network lifetime in [8] and [9], the sink is moved with an adaptive strategy, which is hard to apply. In [4], repositioning of the sink node to enhance the performance metrics is investigated. There is a little work done on the multiple-sink wireless sensor networks. In [11], multiple sink location problems to manage the energy efficiently and solutions to these problems are presented. In [12], the formulation to find optimal locations of multiple sinks is proposed. Reference [3] proposes a solution for correlated data gathering to minimize 
the system-wide energy consumption. In [1], the worst case analysis of sensor networks with multiple sinks, namely, network calculus is presented. Reference [10] presents a methodology for optimally designing the topology to optimize the communication cost for wireless sensor networks with multiple sinks. Reference [2] proposes a model to adopt existing single-sink algorithms to multi-sink networks. Reference [13] proposes a two-tier data dissemination approach for large-scale sensor networks, which is completely proactive and energy-inefficient.

\section{MOBILE MULTIPLE SINKS IN LARGE-SCALE WIRELESS SENSOR NETWORKS}

We use the Stateless Weight Routing with Multiple Sinks (MS-SWR) algorithm [14] for routing. We show that MSSWR can be used in an environment where the sinks are mobile. Actually, MS-SWR has a natural adaptive structure to mobile environments. We explain this property in the following paragraphs. MS-SWR is a distributed stateless routing algorithm that does not require a priori topology information. It can be used with any number sink nodes without any modification in the routing protocol. MS-SWR protocol is based on the Stateless Weight Routing (SWR) protocol [15]. SWR is a new novel stateless and beaconless routing algorithm for wireless sensor and ad hoc networks. In SWR, routing is completely achieved at network layer rather than a cross-layered i.e. MAC-involved solution. Since no routing table is kept and no beaconing has to be done, it can be called a reactive stateless protocol.
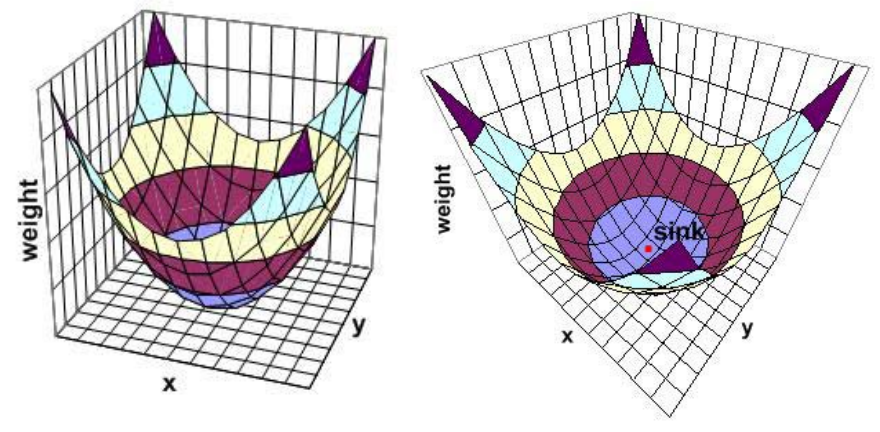

Fig. 1 Weight metric provides a natural flow toward the sink.

SWR uses weight values instead of geographical positions in routing decisions. Each node derives its weight value dynamically from its current position and also it may involve some QoS (Quality of Service) parameters such energy left at the node. Nodes away from the sink node usually has greater weight values with respect to closer ones, as the sink has a weight value 0 [15]. A weight diagram is shown in Fig. 1, where only one sink is positioned in the center of the operation area. Therefore, the routing algorithm has a natural data flow toward the sink. Hence, the use of weight metric makes the routing process simple and minimizes delay, energy consumption, and processing requirements at nodes in routing decision phase. In SWR, when a node has data to transmit, inserts its and the destination's weight values into the packet, and broadcasts the packet. When a node receives a packet, it compares its own weight value with the weight values in the packet. If its weight value is between the transmitting node's weight value and the destination's weight value, it rebroadcasts the packet, or drops the packet otherwise.

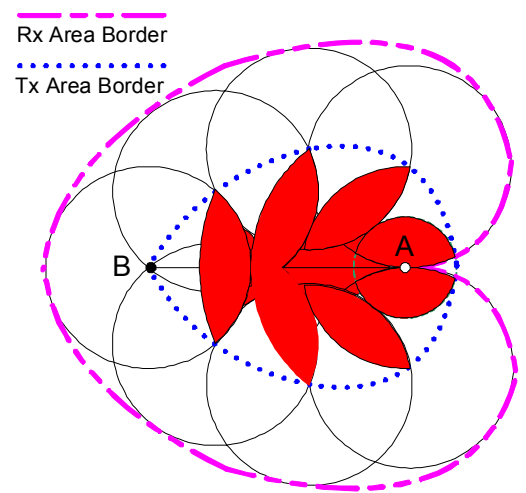

Fig. 2. Possible transmission and receive areas between the source node $B$ and destination node $A$.

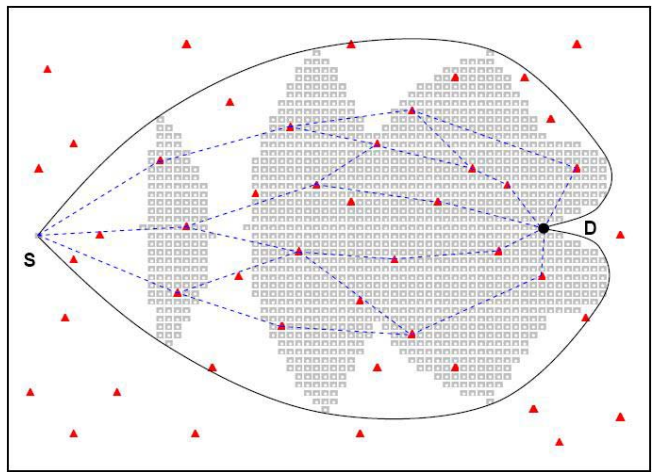

(a)

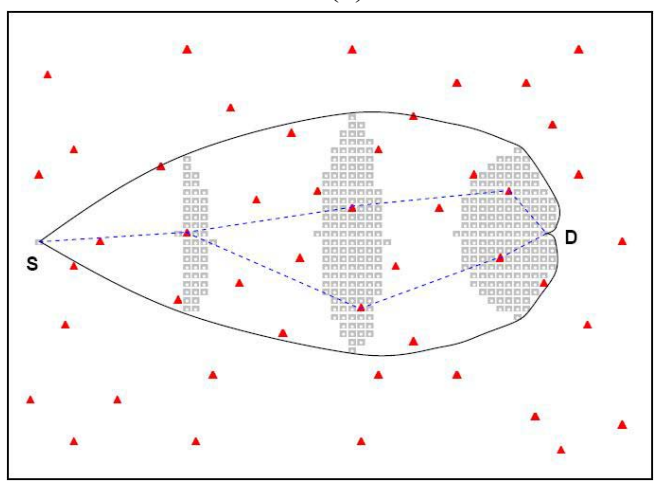

(b)

Fig. 3 Threshold usage in MS-SWR determines the number of multiple-paths. With a higher threshold value in (b), fewer nodes relay the data constructing fewer paths than (a). 


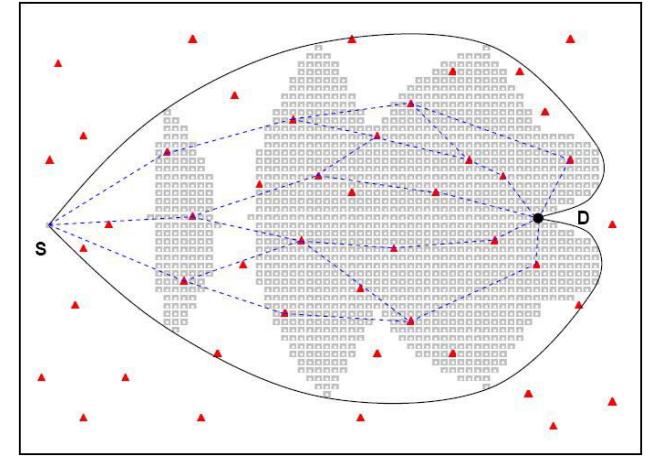

(a)

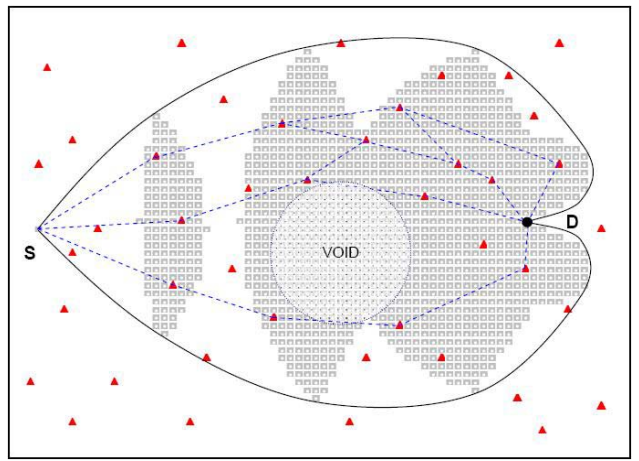

(b)

Fig. 4. Constructed multiple paths in MS-SWR may eliminate voids implicitly.

The derived algorithm in [15] constructs multiple paths. Possible rebroadcasting nodes remain in the symmetric pedal curve shaped transmission area as shown Fig. 2. Such an area between the source and the destination is constructed but when the distance between the source and the sink is far enough. To limit the number of rebroadcasting nodes and the number of multiple paths, a threshold value is used (Fig. 3). Usage and effects of the threshold value and the multiple path construction criteria are defined in [16]. Constructed multiple paths present an implicit void avoidance characteristic since the multiple-paths may provide the data to flow to the destination even to the voids (Fig. 4). In [16], also an explicit void elimination algorithm is presented for SWR, where the implicit way becomes unsuccessful. Void avoidance algorithm guarantees the delivery of the data to the destination, if there is any existing path.

As the network size grows, it is essential to use multiple sinks for partitioning the operation area. The question arises whether the used routing can be applied when the network size grows and/or number of sinks increases. As defined in [14], MS-SWR protocol can be implemented in a network with any number of sinks. Number of the sinks does not affect the protocol. A sink node informs the other nodes in the network about its position. Therefore, nodes choose the closest sink as destination. Then, nodes calculate their weight values with respect to the selected sink node. Situation is depicted in Fig. 5, which is the multiple sink case of Fig. 1.
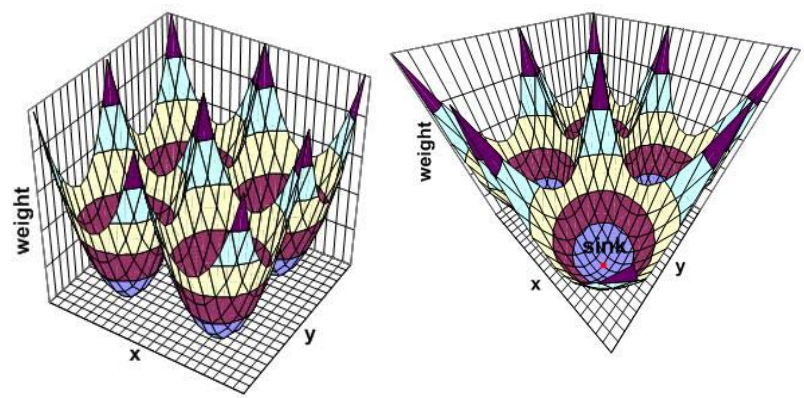

Fig. 5 Multiple sink usage. Nodes choose the closest sink and calculate their own weights with respect to the selected sink.

\section{A. Sinks' Mobility}

Multiple sink usage in large scale networks is investigated in [14]. In [14], it is shown that when the SWR protocol is applied, multiple sink usage decreases the energy consumption in routing processes and contributes to prolong the lifetime of the network. However, it is also show that GPRS and flooding do not exhibit the same performance enhancements without modification. Performance enhancement in the MS-SWR with respect to multiple-sink usage is related with the data flow approach presented in [15]. As the distance between the source node and the sink node becomes shorter, the number of retransmitting nodes (relay nodes) decreases. Therefore, energy consumption is reduced as the number of sinks increases. More energy can even be saved by introducing mobile sinks to the system. The essential approach is again to decrease the distance between source nodes and the sink nodes.

As known, in wireless sensor network applications, sensor nodes acquire environmental data and send it to the sink. This may be done either as a periodic acquisition or an event triggered acquisition process. Some surveillance and detection systems are considered as event triggered acquisition process in which a group of nodes in the event area, which are close to each other may encounter the same event and report that event to the sink. We call these nodes as EAR (Event Area) nodes. Upon an event, EAR nodes start sending data to sink. For the same event, multiple transmissions occur simultaneously causing multiple copies of the data from different sources to flow toward the sink. We can decrease the number of transmissions by shortening the paths if we move the sink toward the EAR nodes. The movement of the sink should be limited to make only a few steps toward EAR nodes in order not to break the original deployment strategy.

Besides that, if there is a void on the path toward the sink and if the sink works out the existence of such a void by the help of the received packets, a movement to eliminate the 
void problem may be considered. However, this is an optimization problem that is out of the scope of this paper.

The number of hops for the sink's movement depends on the number of total sinks in the network (i.e., the number of sensor nodes per sink) and the average distance between source nodes and the sink node. When a sink realize that received data is related to the same event, it moves toward the direction of the source node. Sink nodes are able to compute the distance of the source node by using the weight value in the header of the packet. We also assume that upon an event the location information of the sensor node is inserted in the first event packet in order to inform sink on it direction. Note that location information will not be transmitted in the further stages of the communication. However distance can be computed with the help of the weight. Regarding to the weight value, movement of the sink may vary from a small step to a large one. The sink informs the other nodes about its new position by a broadcast if it moves significantly, i.e. more than the length of a hop. If the movement is not significant, less than a hop, there is no need to inform the rest of the network.

\section{SIMULATION AND RESULTS}

\section{A. Simulation Parameters}

In this section we present our simulation results. There is no packet loss due to transmission collisions in the simulation environment. To provide the double range property [17], nodes have a sensing range $\left(R_{s}\right)$ of 50 meters and a transmission range $\left(R_{c}\right)$ of 100 meter $\left(R_{c} / R_{s}=2\right)$. The network is designed with the methodology defined in [18] and nodes randomly generate packets with a probability of $0.05 \mathrm{pkt} / \mathrm{min}$. Default threshold value is set to $R_{c} / 2$ for the MS-SWR protocol. The given results are the averages of 10 runs. We assume that packet lengths and energy consumptions at receive and transmit states are identical.

MS-SWR is a reactive stateless geographical routing protocol working independent from the MAC-layer that is used underneath. However, all the other reactive stateless geographical routing protocols propose MAC-layer involved solutions for energy-efficiency in routing. Therefore, we compare the proposed approach with "GPSR without perimeter" algorithm [19] which is proactive stateless geographical routing protocol. Parameters for GPSR are obtained from the results of [20]. GPSR uses periodic beaconing and keeps local topology knowledge proactively. To compare our proposed approach with a reactive protocol, we use flooding. We focused on the energy consumption.

We worked on two scenarios to evaluate the performance of the approach. In the first scenario, a small scale network is constructed with fifty sensor nodes and varying number of stationary sinks. We observed the system-wide energy consumption in routing processes in the network according to the applied protocols. 1000 joule is given to each node for routing processes. Energy consumptions other than in routing processes are not observed.
In the second scenario, a large scale network is constructed with 1600 stationary sensor nodes over $2000 \mathrm{~m}$ x 2000m area. To limit the number of sink nodes to $1 \%$ of the sensor nodes, we deployed 16 mobile sinks. Destination (sink) nodes are positioned uniformly in the operation area. Sensor nodes are stationary while the sink nodes can move in their regions. Sink nodes make their movements to shorten the distance between themselves and the EAR nodes. On a source node's transmission the EAR nodes are forced to make new transmissions for the same data toward the same destination to provide the situation described in Section 3.A. In scenario 2 , we observed the effects of mobility to energy consumption in routing process.

\section{B. Energy Consumption}

Fig. 6 shows the system-wide consumed energy values in routing process with a single sink in scenario 1. GPSR protocol and the flooding algorithm deplete the allocated energy very quickly. In GPSR, the simulation ends after 139 seconds failing to find routes. The overall system energy of the GPSR protocol is a little better than flooding, causing the system to live longer than flooding. GPSR depletes most of its energy at the beaconing, while the flooding depletes its energy on routing process. The observed system energy in GPSR protocol is according to the beaconing period with 1 sec. The system will live longer in GPSR protocol when the beaconing interval is extended. MS-SWR protocol continues to live when the simulation ends after $900 \mathrm{sec}$. In MS-SWR, the energy is consumed only in routing processes..

We observed that increasing the number of the sinks does not affect the performance of the flooding protocol and the GPSR protocol. Adding more sinks does not reduce the transmissions in flooding. On the other hand, in GPSR, increase in the number of the sinks only reduces the number of transmissions in data packets. In GPSR, the energy is mainly consumed in beaconing. Decreasing the shortest path in GPSR only avoids a few transmissions makes no sense in energy consumption. However, in MS-SWR, shortening the distance between the source node and the sink node decreases the number of retransmitting nodes. Therefore, usage of multiple sinks in MS-SWR cause a notable energy consumption decrease in routing process (Fig. 7).

Mobile sinks' performance is shown in Fig. 8 and Fig. 9. In Fig. 8, effect of shortening the path to energy consumption in routing process is investigated. Path length is the hop count between the sink node and the center of the EAR nodes. As sink node gets closer to the EAR nodes, energy gain in routing process increases. In 2-hops path, decreasing the path one hop causes a $60 \%$ energy gain in routing process. And, in 6 -hops path, decreasing the path one hop causes a $27.5 \%$ energy gain in routing process.

Energy consumption comparison between the stationary sink nodes and mobile nodes in routing process is shown in Fig. 9. It seen that using mobile sinks reduces the energy consumption in a considerable amount. 


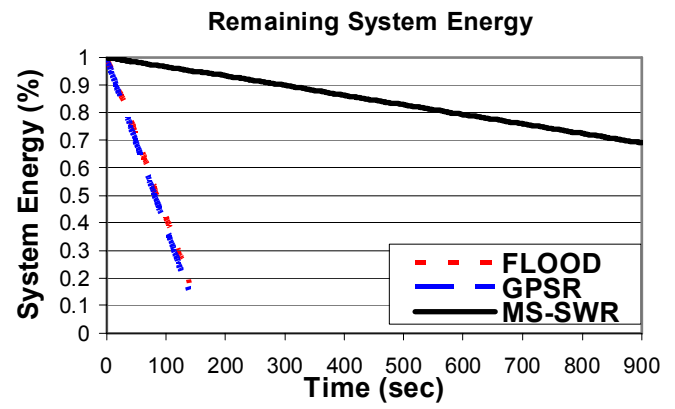

Fig. 6 Remaining energy levels of the protocols in Scenario 1 with a single sink.

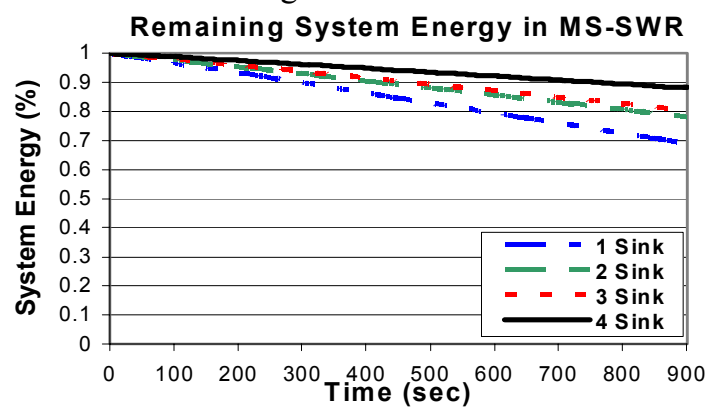

Fig. 7 Remaining system energies of the network when the MS-SWR protocol is used with multiple sinks in Scenario 1.

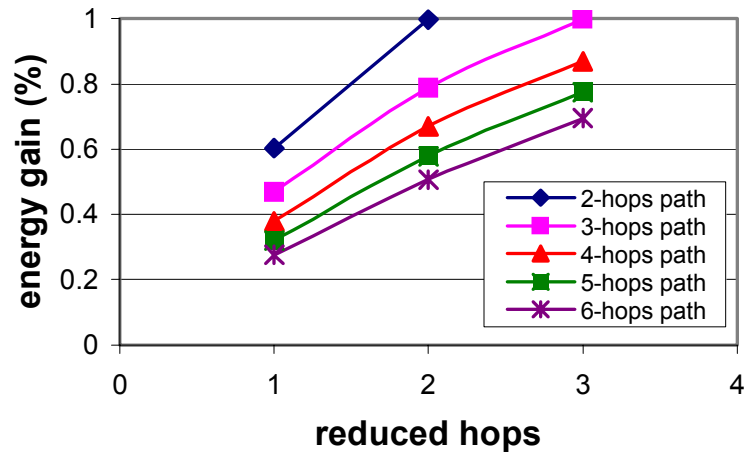

Fig. 8 Reducing the number of hops between the source and the sink node reduce the energy consumption in MSSWR in routing process.

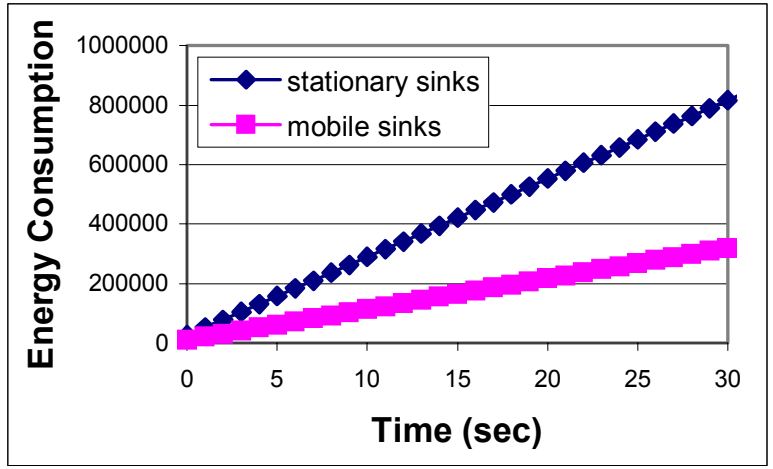

Fig. 9 Mobile sinks reduces the system-wide energy consumption in routing processes in MS-SWR protocol.

\section{V.CONCLUSION}

Energy is the most critical resource in wireless sensor networks and must be used carefully. One approach to decrease the energy consumption is to use mobile sink nodes to collect data. There are some studies which use a mobile sink node to enhance the energy consumption and therefore to prolong the lifetime of the network. However, studies are limited to small scale networks with a single sink node. For large scale wireless sensor networks, we propose to use mobile sinks with MS-SWR protocol to reduce the energy consumption in routing and to extend the lifetime of the network. MS-SWR protocol is a scalable protocol for large size networks. We show that mobile sinks usage enhances the energy related performance metrics.

\section{REFERENCES}

[1] J.B. Schmitt, F.A. Zdarsky, U.Roedig. "Sensor Network Calculus with Multiple Sinks", In Proceedings of the 11th IFIP International Conference on Personal Wireless Communications (PWC'06), Albacete, Spain. Springer LNCS, September 2006.

[2] A. Das, D. Dutta. "Data Acquisition in Multiple-sink Sensor Networks", Mobile Computing and Communications Review 9(3): 82-85 (2005)

[3] K. Yuen, B. Liang, B. Li. "A Distributed Framework for Correlated Data Gathering in Sensor Networks", IFIP 2006

[4] Akkaya, M. Younis, M. Bangad, "Sink Repositioning for Enhanced Performance in Wireless Sensor Networks". Elsevier Computer Networks Journal Vol.49/4 (2005) 512-534

[5] I. Chatzigiannakis, A. Kinalis, S. Nikoletseas. "Sink Mobility Protocols For Data Collection in Wireless Sensor Networks", Proceedings of The International Workshop On Mobility Management and Wireless Access, MobiWac '06 pp 52-59 (2006)

[6] J. Hubaux and J. Luo, "Joint Mobility and Routing for Lifetime Elongation in Wireless Sensor Networks", in IEEE INFOCOM, 2005

[7] J. Luo, J. Panchard, M. Piorkowski, M. Grossglauser,J.P. Hubaux. "MobiRoute: Routing towards a Mobile Sink for Improving Lifetime in Sensor Networks", IEEE/ACM DCOSS San Francisco, California, USA, June 2006.

[8] Z. Vincze, R. Vida."Multi-hop Wireless Sensor Networks With Mobile Sink", Proceedings of the 2005 ACM Conference on Emerging Network Experiment and Technology, pp. 302 - 303, (2005)

[9] Z. Vincze, D. Vass, R. Vida, A. Vidács, A. Telcs. "Adaptive Sink Mobility in Event-Driven Multi-hop Wireless Sensor Networks", Proceedings of the First International Conference on Integrated Internet Ad Hoc and Sensor Networks, InterSense '06, May 2006

[10]M. Kalantari, M. Shayman. "Design Optimization of Multi-Sink Sensor Networks by Analogy to Electrostatic Theory", WCNC 2006. 
[11]E.I. Oyman, C. Ersoy. "Overhead Energy Considerations for Efficient Routing in Wireless Sensor Networks", Computer Network, Vol.46, pp. 465-478, 2004

[12]H. Kim, Y. Seok, N. Choi, Y. Choi, T. Kwon: “Optimal Multi-sink Positioning and Energy-Efficient Routing in Wireless Sensor Networks." ICOIN 2005: 264-274

[13]H. Luo, F. Fe, J. Cheng, S. Lu, L. Zhang. " TTDD: A Two-Tier Data Dissemination Model for Large-scale Wireless Sensor Networks", ACM/Kluwer Mobile Networks and Applications (MONET), Special Issue on ACM MOBICOM

[14]M. Soyturk, T.Altilar. "A Novel Stateless EnergyEfficient Routing Algorithm for Large-Scale Wireless Sensor Networks with Multiple Sinks", IEEE WAMICON'06, Dec. 2006.

[15]M. Soyturk, T.Altilar. "Source-Initiated Geographical Data Flow for Wireless Ad Hoc and Sensor Networks", IEEE WAMICON'06, Dec. 2006.
[17]C. Avin. "Fast and Efficient Restricted Delaunay Triangulation in Random Geometric Graphs", Workshop on Combinatorial and Alg. Aspects of Networking, 2005

[18]Y. Yu, B.Hong, V.K. Prasanna. "On Communication Models for Algorithm Design in Networked Sensor Ssytems: A Case Study", Elsevier Pervasive and Mobile Computing Journal, Vol.1, No:1, 95-122, Mar. (2005)

[19]B. Karp, H.T. Kung, “GPSR: Greedy Perimeter Stateless Routing for Wireless Networks". Mobicom 2000, (2000)

[20]D. Son, A. Helmy, B. Krishnamachari. "The Effect of Mobility-Induced Location Errors on Geo.Routing in Mobile Ad Hoc and Sensor Networks: Analysis and Improvement Using Mobility Prediction", IEEE Trans. Mobile Comput.Vol.3, No.3, Jul-Sep, 233-245 (2004)

[16]M. Soyturk, T.Altilar. "Stateless Data Flow Approach with Void Avoidance for Wireless Ad Hoc and Sensor Networks", International Symposium on Wireless Pervasive Computing, Feb. 2007. 